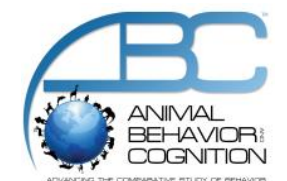

\title{
Perception and Misperception of the Animal's "Self-World": Introduction to the Special Issue on Comparative Perception
}

\author{
Audrey E. Parrish ${ }^{1 *}$ and Christian Agrillo ${ }^{2}$
}

\author{
${ }^{1}$ The Citadel, Charleston, South Carolina, USA \\ ${ }^{2}$ University of Padova, Padova, Italy \\ *Corresponding author (Email: audrey.parrish1@gmail.com)
}

Citation - Parrish, A. E., \& Agrillo, C. (2017). Perception and misperception of the animal's "self-world": Introduction to the special issue on comparative perception. Animal Behavior and Cognition, 4(3), $200-203$. https://doi.org/10.26451/abc.04.03.01.2017

We are excited to bring the readers of Animal Behavior and Cognition a diverse group of research projects that share the common theme of better understanding how animals, including humans, perceive, misperceive, and ultimately interact with their physical and social worlds. The 13 articles contained herein represent a wide variety of taxa and topics that are currently explored within the sub-field of comparative perception. Jakob von Uexküll's (1934/1957) concept of the Umwelt is highlighted throughout this Special Issue, with clever research designs aimed to take us inside the inner-worlds of several species including representatives from insects, fish, reptiles, birds, and mammals. The Umwelt, or idea of a species-unique self-world, is well-known within comparative psychology and ethology, and researchers that work with animals are challenged with tapping into minds that sense, perceive, categorize, and interact with their external worlds in drastically different and complex manners (e.g., visually-orienting primates vs. echolocating bats and dolphins vs. the infamous chemical and temperaturesensing ticks as originally described by Uexküll, 1934/1957). Uncovering the ways in which other species perceive their worlds is not a new endeavor, and comprehensive reviews of comparative perception from a biological, psychological, and neurological standpoint can be found elsewhere (e.g., see Lazareva, Shimzu, \& Wasserman, 2012). The goal of the current Special Issue is to highlight recent experimental work in comparative perception including the study of basic visual abilities of animals via perception and discrimination tasks, categorization, facial processing, and illusory perception studies.

In the first series of papers, basic perceptual and discrimination abilities of a variety of animals are explored. For example, Adams et al. assessed the limits of spatial vision in Sumatran orangutans (Pongo abelii) using a contrast sensitivity task. This work extends research on spatial vision to an additional nonhuman primate species that is used often as a model of great ape cognition. In that same vein, Fuss et al. examined perception and discrimination of simple and complex movement in juvenile grey bamboo sharks (Chiloscyllium griseum) revealing sensitivity to biological motion in a variety of settings and with a range of stimuli. In an additional study of basic perceptual abilities, Chiandetti and 
Caputi assessed visual shape recognition in crayfish (Procambarus clarkii) using a habituation procedure. These results revealed that crayfish use visual information to discriminate stimuli in addition to a reliance on other sensory input including tactile information and chemoreception. Other research teams explored whether there is differential sensitivity to various stimulus dimensions. Lazareva and Share reported that human adults and pigeons (Columba livia) are more sensitive to size than speed using a two-alternative simultaneous discrimination task, with faster learning and higher sensitivity to size discrepancies relative to comparable differences in speed. Similarly, Sturz et al. used psychophysics and eye-tracking procedures to determine the geometric cues that human adults rely upon to perceive shapes including squares and rectangles. Implications of geometrical processing and spatial cognition are discussed. Establishing the degree to which the visual systems of closely- and distantly-related species overlap is imperative to their inclusion in additional perceptual, cognitive, and biological studies and furthers our understanding of the evolutionary continuity of nonhuman and human visual systems.

Categorization is a highly important cognitive process by which individuals integrate perceptual and attentional abilities to identify and classify stimuli that are similar to one another. Many animals learn to classify visual stimuli according to their unique features and this is represented in Herbranson et al.'s work investigating how pigeons learn to classify complex visual stimuli. Pigeons learned to categorize sets of moving stimuli based upon angle of motion and speed (or both), as well as categorize non-moving stimuli based upon size but not orientation angle. These results revealed that, for pigeons, angle as a direction of travel is more salient than angle of orientation in static stimuli. These results are discussed in light of species differences with humans and the ecological pressures that lead to adaptive differences in cognitive processes including visual perception and categorization.

Perceptual processing of social cues in addition to nonsocial stimuli in an animal's physical world also is highly adaptive, including potentially locating, identifying, and interacting with other individuals such as kin, competitors, mates, predators, and prey. An individual's ability to perceive and monitor the spatial distribution and location of conspecifics in their vicinity is one way by which perception is key to social cognition. For example, in their paper, Mercado et al. explored the use of acoustic cues in the songs of black-capped chickadees (Parus atricapillus). Song information enabled the listener to monitor the distance, movement, and interaction of other chickadees. Beyond the perception of acoustic cues, the ability to visually discriminate individual faces of conspecifics and even other species is adaptive to a wide range of social species. Aside from humans, a growing body of research suggests that faces are processed similarly by some species such as primates (e.g., see Parr, 2011, for a review) but also that other distantly-related species including some insect species are perhaps capable of processing facial stimuli as well (e.g., Sheehan \& Tibbetts, 2011). Two papers address facial processing in the current Special Issue, including Avarguès-Weber et al.'s report on free flying wasps' (Vespula vulgaris) facial recognition abilities of human faces using an appetitive-aversive differential conditioning procedure. Avarguès-Weber et al. suggest that a general visual system may be equipped to address complex recognition tasks, even those that involve conspecific and perhaps heterospecific facial recognition. Beran et al. presented unique images to pre-school children, rhesus macaques (Macaca mulatta), and capuchin monkeys (Sapajus apella) that resemble human faces when presented to human adults but are comprised of fruits, vegetables, and other objects rather than anatomically correct features (e.g., eyes, nose, mouth, etc.). The role of perceptual organization and processing mode (the use of global vs. local cues) and its effect on facial processing in primates are discussed, in light of the finding that monkeys are less likely than children to see faces in these non-face stimuli.

The processing of social information by the brain also is explored in this Special Issue.

Specifically, lateralized eye use is suggested to increase brain efficiency by divvying up the processing of different types of visual information within each cerebral hemisphere (social information, movement, emotion, etc.). Lateralization has been extended to a wide range of animal species and Frohnwieser et al. continue this work with bearded dragons (Pogona vitticeps). In their paper, they assessed lateralization by presenting male and female bearded dragons with video stimuli of moving or stationary social conspecifics and prey. Sex differences are discussed in terms of left and right eye biases for social stimuli as well as the role of motion perception in lateralized eye use. 
The final group of articles in this Special Issue concerns the ways in which animals not only perceive their world accurately, but also how they misperceive external stimuli. A growing body of literature addresses the role of illusion perception among nonhuman animals, in which perception does not accurately reflect an animal's physical world (e.g., Barbet \& Fagot, 2002; Fujita, 1997; Fujita, Blough, \& Blough, 1991; Nakamura, Watanabe, \& Fujita, 2008, 2014; Parrish, Brosnan, \& Beran, 2015; Pepperberg, Vicinay, \& Cavanagh, 2008; Rosa Salva, Rugani, Cavazzana, Regolin, \& Vallortigara, 2013; Sovrano \& Bisazza, 2009; Suganuma, Pessoa, Monge-Fuentes, Castro, \& Tavares, 2007; see Agrillo, Petrazzini, \& Dadda, 2013, Mascalzoni \& Regolin, 2011, and Wasserman, 2012 for reviews). Visual illusions are an increasingly popular tool used to uncover the mechanisms by which a highly functional perceptual system operates. For example, many illusions emerge as a byproduct of an otherwise efficient rule-based system that relies upon certain perceptual organization laws (global-precedence vs. localprecedence and Gestalt principles) or one that is shaped via prior experiences. Cross-species similarities in illusion perception shed further light on evolutionary continuity, and thus misperceptions can provide critical insight to the functioning of a perceptual system and its supporting mechanisms. In the current Special Issue, several authors report on illusory phenomena in a range of species, including expansioncontraction color effects in redtail splitfin fish (Xenotoca eiseni, Albertazzi et al.) and the geometric Delboeuf size illusion in ring-tailed lemurs (Lemur catta, Santacà et al.). Additionally, Pepperberg provides a review of optical illusions in African grey parrots (Psittacus erithacus), including the MüllerLyer Illusion and amodal and modal perception. These authors all employ a variety of methodological approaches to the study of illusory perception, including the observation of spontaneous behavior or after operant conditioning procedures in two-choice discrimination tasks and the use of referential speech by parrots to indicate their response in an experimental setting. Important differences in choice procedure, training techniques, and stimuli design are considered within these articles. Together, these and other comparative studies of illusory perception provide an alternate means by which we can investigate the perceptual system of other species, including evolutionary-distant species such as fish, and better understand the rules that govern these systems.

Investigating how animals see the world in ways like and unlike humans' perception of the world has interested philosophers, biologists and psychologists alike for a long time. There are indeed countless separate Umwelts or subjective worlds available for exploration on the same physical planet. Researchers working in comparative perception are explorers of these unique worlds, searching for a glimpse into the minds of other species through the individuals who serve as ambassadors for their species. We hope that readers will agree that the collection of papers included in this Special Issue further our efforts in this endeavor.

\section{Acknowledgements}

We are deeply indebted to the authors who have contributed their unique works to this Special Issue, as well as the editors of Animal Behavior and Cognition and the many reviewers who volunteered their time and expertise.

\section{References}

Agrillo, C., Miletto Petrazzini, M.E., \& Dadda, M. (2013). Illusory patterns are fishy for fish, too. Frontiers in Neural Circuits, 7, 137.

Barbet, I., \& Fagot, J. (2002). Perception of the corridor illusion by baboons (Papio papio). Behavioural Brain Research, 132, 111-115.

Fujita, K. (1997). Perception of the Ponzo illusion by rhesus monkeys, chimpanzees, and humans: Similarity and difference in the three primate species. Perception and Psychophysics, 59, 284-292.

Fujita, K., Blough, D.S., \& Blough, P.M. (1991). Pigeons see the Ponzo illusion. Animal Learning and Behavior, 19, 283-293.

Lazareva, O. F., Shimizu, T., \& Wasserman, E. A. (Eds.). (2012). How animals see the world: Comparative behavior, biology, and evolution of vision. New York: Oxford University Press. 
Mascalzoni, E., \& Regolin, L. (2011). Animal visual perception. Wiley Interdisciplinary Reviews: Cognitive Science, 2, 106-116.

Nakamura, N., Watanabe, S., \& Fujita, K. (2008). Pigeons perceive the Ebbinghaus-Titchener circles as an assimilation illusion. Journal of Experimental Psychology: Animal Behavior Processes, 34, 375-387.

Nakamura, N., Watanabe, S., Fujita, K. (2014) A reversed Ebbinghaus-Titchener illusion in bantams (Gallus gallus domesticus). Animal Cognition, 17, 471-481

Parr, L. A. (2011). The evolution of face processing in primates. Philosophical Transactions of the Royal Society B: Biological Sciences, 366, 1764-1777.

Parrish, A. E., Brosnan, S. F., \& Beran, M. J. (2015). Do you see what I see? A comparative investigation of the Delboeuf illusion in humans (Homo sapiens), rhesus monkeys (Macaca mulatta), and capuchin monkeys (Cebus apella). Journal of Experimental Psychology: Animal Learning and Cognition, 41, 395-405.

Pepperberg, I. M., Vicinay, J., \& Cavanagh, P. (2008). Processing of the Müller-Lyer illusion by a grey parrot (Psittacus erithacus). Perception, 37, 765-781.

Rosa Salva, O., Rugani, R., Cavazzana, A., Regolin, L., \& Vallortigara, G. (2013). Perception of the Ebbinghaus illusion in four-day-old domestic chicks (Gallus gallus). Animal Cognition, 16, 895-906.

Sheehan, M. J., \& Tibbetts, E. A. (2011). Specialized face learning is associated with individual recognition in paper wasps. Science, 334, 1272-1275.

Sovrano, V. A., \& Bisazza, A. (2009). Perception of subjective contours in fish. Perception, 38, 579-590.

Suganuma, E., Pessoa, V. F., Monge-Fuentes, V., Castro, B. M., \& Tavares, M. C. H. (2007). Perception of the Müller-Lyer illusion in capuchin monkeys (Cebus apella). Behavioural Brain Research, 182, 67-72.

Uexküll's, J. von. (1957). A stroll through the worlds of animals and men: A picture book of invisible worlds. In C. H. Schiller (Ed.), Instinctive behavior: The development of a modern concept (pp. 5-80). New York: International Universities Press. (Original work published 1934).

Wasserman, E. A. (2012). Illusory perception in animals: Observations and interpretations. In O. F. Lazareva, T. Shimizu \& E. A. Wasserman (Eds.), How animals see the world: Comparative behavior, biology, and evolution of vision (pp. 93-99). New York: Oxford University Press. 